\title{
An agile FCM for real-time modeling of dynamic and real-life systems
}

\begin{abstract}
Fuzzy cognitive map (FCM) is a well-established model of control and decision making based on neural network and fuzzy logic methodologies. It also serves as a powerful systematic way for analyzing real-life problems where tens of known, partially known, and even unknown factors contribute to complexity of a system. FCM-based inference requires a neural activation function much like other neural network systems. In modeling, in addition to an activation function, FCM involves with weight training to learn about relationships as they exist among contributing factors. Therefore, numerous contributing factors could be analyzed to understand the behaviors of factors within a real-life system and to represent it in form of tangible matrices of weights. This article discusses a new incremental FCM activation function, named cumulative activation, and introduces a new weight training technique using simulated annealing (SA) known as agile FCM. Smooth variation of FCM nodes that is due to cumulative nature of inference results into faster convergence, while a unique minimum cost solution is guaranteed using the SA training module that is entirely expert-independent. A combination of these two techniques suits time-related applications where inclusion of temporal features is necessary. The resulted system is examined through numerical example datasets where the candidate FCM shows sensitivity to dynamic variables over time. A real-life example case is included as well to further support the effectiveness of the developed FCM in modeling of natural and complex systems.
\end{abstract}

Keyword: Temporal fuzzy cognitive map; Real-time response; Relationship modeling 